\title{
On the connection between Gaussian statistics and excited-state linear response for time-dependent fluorescence
}

\author{
Brian B. Laird ${ }^{\text {a) }}$ and Ward H. Thompson ${ }^{\text {b) }}$ \\ Department of Chemistry, University of Kansas, Lawrence, Kansas 66045
}

(Received 9 January 2007; accepted 14 May 2007; published online 6 June 2007)

\begin{abstract}
Time-dependent fluorescence (TDF) of a chromophore in a polar or nonpolar solvent is frequently simulated using linear-response approximations. It is shown that one such linear-response-type approximation for the TDF Stokes shift derived by Carter and Hynes [J. Chem. Phys. 94, 5961 (1991)] that is based on excited-state dynamics gives the same result as that obtained by assuming Gaussian statistics for the energy gap. The derivation provides insight into the much discussed relationship between linear response and Gaussian statistics. In particular, subtle but important differences between the two approximations are illuminated that suggest that the result is likely more generally applicable than suggested by the usual linearization procedure. In addition, the assumption of Gaussian statistics directly points to straightforward checks of the validity of the approximation with essentially no additional computational effort. (C) 2007 American Institute of Physics. [DOI: 10.1063/1.2747237]
\end{abstract}

A common experimental approach for probing solvation dynamics relevant to charge-transfer processes is the measurement of the time-dependent fluorescence Stokes shift. ${ }^{1-4}$ In these experiments a probe dye molecule - generally with a charge-transfer electronic transition that is accompanied by a significant change in dipole moment-is excited and the fluorescence energy $\Delta E(t)$ is measured as a function of the time after excitation. Typically, the results are plotted as the normalized dynamic Stokes shift $S(t)$,

$$
S(t)=\frac{\Delta E(t)-\Delta E(\infty)}{\Delta E(0)-\Delta E(\infty)},
$$

where $\Delta E(\infty)$ is the relaxed Stokes shift. The decay of $S(t)$ with time provides information about time scales for solvent reorganization, time scales that are relevant, for example, to electron and proton transfer reactions.

The normalized dynamic Stokes shift can be simulated using nonequilibrium molecular dynamics. In this approach, an equilibrium molecular-dynamics trajectory of the groundstate dye molecule in solution is run to generate a representative set of initial conditions for excitation. Each of these initial configurations of the solute and solvent nuclei is used as the starting point of a nonequilibrium trajectory with the dye electronic state changed to the excited one; this creates an excited-state dye molecule in a nonequilibrium solvation configuration. The fluorescence energy $\Delta E(t)$ is then followed during the subsequent nonequilibrium trajectory and is used to compute $S(t)$. Thus, this simulation is quite analogous to the experiment.

Another approach to simulating the results of timedependent fluorescence measurements is to invoke the linearresponse approximation. In this case, the dynamic Stokes shift can be approximated by results from equilibrium

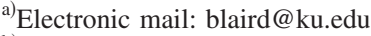

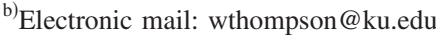

molecular-dynamics simulations. The general approach is to write the Hamiltonian for the system as a zeroth-order Hamiltonian and a "perturbation" that is turned on at time $t=0$ (the moment of excitation),

$$
H(t)=H_{g}+\theta(t)\left[H_{e}-H_{g}\right],
$$

where $\theta(t)$ is the Heaviside step function and $H_{g}$ and $H_{e}$ are the Hamiltonians for the ground- and excited-state dye molecule, respectively. The perturbation, however, is just the fluorescence energy,

$$
\Delta E=H_{e}-H_{g} .
$$

It is sometimes convenient to write the perturbation instead in terms of a variable $\Delta \varepsilon$ defined by

$$
\Delta \varepsilon=H_{e}-H_{g}-\langle\Delta E\rangle_{g},
$$

where

$$
\langle\cdot\rangle_{g}(t)=\frac{\int d \boldsymbol{\Gamma} \cdot e^{-\beta H_{g}}}{\int d \boldsymbol{\Gamma} e^{-\beta H_{g}}},
$$

with $\boldsymbol{\Gamma}=(\mathbf{p}, \mathbf{q})$. However, either choice leads to fundamentally the same results.

The key quantity in the time-dependent fluorescence is the nonequilibrium energy gap,

$$
\begin{aligned}
\langle\Delta E\rangle_{\mathrm{ne}}(t) & =\frac{\int d \boldsymbol{\Gamma} \Delta E(t) e^{-\beta H_{g}}}{\int d \boldsymbol{\Gamma} e^{-\beta H_{g}}}, \\
& =\frac{\int d \boldsymbol{\Gamma}\left\{e^{-\hat{L}_{e} t} \Delta E(0)\right\} e^{-\beta H_{g}}}{\int d \boldsymbol{\Gamma} e^{-\beta H_{g}}} .
\end{aligned}
$$

Note that this requires initial conditions based on the groundstate equilibrium distribution followed by dynamics on the excited state. Two approaches to approximating this nonequilibrium average are based on (1) describing the time dependence in the excited state with ground-state dynamics cor- 
rected to first-order in the perturbation, and (2) describing the ground-state distribution using the excited-state distribution corrected to first-order in the perturbation. The former, which is the "traditional" linear-response approach, ${ }^{5}$ gives an approximation to $S(t)$ as $^{6}$

$$
S(t) \approx \frac{\langle\Delta \varepsilon(t) \Delta \varepsilon(0)\rangle_{g}}{\left\langle\Delta \varepsilon(0)^{2}\right\rangle_{g}} \equiv C_{g}(t) .
$$

Note that in this linear-response approximation only correlation functions obtained from equilibrium ground-state dynamics are required. It can be shown that if traditional linear response holds, then this is equivalent to an analogous expression with correlation functions evaluated in the excited state, i.e., Eq. (16) below. ${ }^{7}$ However, it is widely appreciated that excited- and ground-state dynamics can often differ appreciably. ${ }^{8-13}$ Such cases are sometimes considered to represent a breakdown of the linear-response approximation, though, for example, Tao and Stratt argue, but do not prove, that a stronger condition is more appropriate-one based on whether the energy gap exhibits Gaussian statistics, since "linear-response predictions are obeyed exactly if the liquid fluctuations obey Gaussian statistics."13 Skaf and Ladanyi found that the linear-response approximation can be unsatisfactory even when the ground- and excited-state correlation functions are the same. ${ }^{14}$ Since the derivation of the excitedstate correlation function from Eq. (8) relies on the linearresponse approximation, it is useful to consider a different approach to the derivation based instead on the second approximation described above. This provides additional perspective on the relation between Gaussian statistics and linear response in the ground and excited states. Moreover, it allows us to address approximations in more complex systems, e.g., nanoconfined solvents for which the solute dye molecule can sit in two different physical locations with different solvation properties in the ground and excited electronic states. ${ }^{11}$ That approach is the focus of this letter.

Instead of approximating the excited-state time dependence in Eq. (7), Carter and Hynes noted that the equation can be rewritten using

$$
e^{-\beta H_{g}}=e^{-\beta H_{e}} e^{\beta \Delta E},
$$

to give

$$
\langle\Delta E\rangle_{\mathrm{ne}}(t)=\frac{\int d \boldsymbol{\Gamma} \Delta E(t) e^{\beta \Delta E} e^{-\beta H_{e}}}{\int d \boldsymbol{\Gamma} e^{\beta \Delta E} e^{-\beta H_{e}}} .
$$

Dividing the numerator and denominator by the excited-state partition function gives

$$
\langle\Delta E\rangle_{\mathrm{ne}}(t)=\frac{\left\langle\Delta E(t) e^{\beta \Delta E}\right\rangle_{e}}{\left\langle e^{\beta \Delta E}\right\rangle_{e}} .
$$

However, if we define $\overline{\Delta E} \equiv\langle\Delta E\rangle_{e}$, this can be rewritten as

$$
\langle\Delta E\rangle_{\mathrm{ne}}(t)=\frac{\left\langle\Delta E(t) e^{\beta \delta \Delta E}\right\rangle_{e} e^{-\beta \overline{\Delta E}}}{\left\langle e^{\beta \delta \Delta E}\right\rangle_{e} e^{-\beta \overline{\Delta E}}}=\frac{\left\langle\Delta E(t) e^{\beta \delta \Delta E}\right\rangle_{e}}{\left\langle e^{\beta \delta \Delta E}\right\rangle_{e}},
$$

where $\delta \Delta E(t)=\Delta E(t)-\overline{\Delta E}$. Subtracting the equilibrium (fully relaxed) fluorescence energy gives

$$
\begin{aligned}
\langle\Delta E\rangle_{\mathrm{ne}}(t)-\langle\Delta E\rangle_{\mathrm{ne}}(\infty) & =\frac{\left\langle\Delta E(t) e^{\beta \delta \Delta E}\right\rangle_{e}-\overline{\Delta E}\left\langle e^{\beta \delta \Delta E}\right\rangle_{e}}{\left\langle e^{\beta \delta \Delta E}\right\rangle_{e}} \\
& =\frac{\left\langle\delta \Delta E(t) e^{\beta \delta \Delta E}\right\rangle_{e}}{\left\langle e^{\beta \delta \Delta E}\right\rangle_{e}} .
\end{aligned}
$$

Following Carter and Hynes, the exponential can then be linearized, using $e^{\beta \delta \Delta E} \simeq 1+\beta \delta \Delta E$, to obtain a "linearresponse-type," or Onsager regression hypothesis, ${ }^{15,16,18}$ approximation,

$$
\begin{aligned}
& \langle\Delta E\rangle_{\mathrm{ne}}(t)-\langle\Delta E\rangle_{\mathrm{ne}}(\infty) \\
& \simeq \frac{\langle\delta \Delta E(t)\rangle_{e}+\beta\langle\delta \Delta E(t) \delta \Delta E(0)\rangle_{e}}{1+\beta\langle\delta \Delta E\rangle_{e}}, \\
& \simeq \beta\langle\delta \Delta E(t) \delta \Delta E(0)\rangle_{e},
\end{aligned}
$$

noting that $\langle\delta \Delta E\rangle_{e}=0$. Thus, the resulting approximation to the normalized dynamic Stokes shift is given by

$$
S(t) \approx \frac{\langle\delta \Delta E(t) \delta \Delta E(0)\rangle_{e}}{\left\langle(\delta \Delta E)^{2}\right\rangle_{e}} \equiv C_{e}(t)
$$

This approximation to $S(t)$ has the advantage that the dynamics determining the energy gap autocorrelation function takes place on the excited state, just as in the nonequilibrium case. As noted above, this expression can also be obtained from Eq. (8) by assuming that traditional linear response holdsi.e., the differences between the ground- and excited-state correlation functions are of higher than linear order. This derivation, however, does not require such an equivalence.

The success of Eq. (16) is interesting since, in general, the truncation of the expansion $\exp \{\beta \delta \Delta E\}$ is not obviously justified in that $\delta \Delta E$ can be (and often is) larger than $k_{B} T$. However, as we show below, the same result can be derived (and better understood) based on the more general assumption that the system exhibits Gaussian statistics. ${ }^{13}$ To see this, we begin with Eq. (13) and note that the numerator can be written as

$$
\left\langle\delta \Delta E(t) e^{\beta \delta \Delta E}\right\rangle_{e}=\sum_{n=0}^{\infty} \frac{1}{n !} \beta^{n}\left\langle\delta \Delta E(t) \delta \Delta E(0)^{n}\right\rangle_{e},
$$

$$
\begin{aligned}
= & \sum_{n=0}^{\infty} \frac{1}{(2 n) !} \beta^{2 n}\left\langle\delta \Delta E(t) \delta \Delta E(0)^{2 n}\right\rangle_{e} \\
& +\sum_{n=0}^{\infty} \frac{1}{(2 n+1) !} \beta^{2 n+1}\left\langle\delta \Delta E(t) \delta \Delta E(0)^{2 n+1}\right\rangle_{e} .
\end{aligned}
$$

If $\delta \Delta E(t)$ is a Gaussian random variable with zero mean $\left(\langle\delta \Delta E(t)\rangle_{e}=0\right.$ for all $\left.t\right)$ then all $m$-point correlation functions $\left\langle\delta \Delta E\left(t_{1}\right) \delta \Delta E\left(t_{2}\right), \ldots, \delta \Delta E\left(t_{m}\right)\right\rangle_{e}$ are equal to zero if $m$ is odd and, if $m$ is even, to the sum of all possible factorizations of the $\delta \Delta E$ in terms of two point correlation functions, ${ }^{19}$ $\left\langle\delta \Delta E\left(t_{i}\right) \delta \Delta E\left(t_{j}\right)\right\rangle_{e}$, a property often referred to as Wick's theorem. ${ }^{20}$ Applying Wick's theorem to the correlation functions in Eq. (18) gives 


$$
\left\langle\delta \Delta E(t) \delta \Delta E(0)^{2 n}\right\rangle_{e}=0,
$$

and

$$
\left\langle\delta \Delta E(t) \delta \Delta E(0)^{2 n+1}\right\rangle_{e}=\frac{(2 n+1) !}{n ! 2^{n}}\langle\delta \Delta E(t) \delta \Delta E(0)\rangle_{e}\left\langle\delta \Delta E^{2}\right\rangle_{e}^{n} .
$$

Using these approximations gives Eq.(18) as

$$
\begin{aligned}
\left\langle\delta \Delta E(t) e^{\beta \delta \Delta E}\right\rangle_{e} & \simeq \beta\langle\delta \Delta E(t) \delta \Delta E(0)\rangle_{e} \sum_{n=0}^{\infty} \frac{1}{n !} \frac{\beta^{2 n}\left\langle\delta \Delta E^{2}\right\rangle_{e}^{n}}{2^{n}}, \\
& \simeq \beta\langle\delta \Delta E(t) \delta \Delta E(0)\rangle_{e} e^{\beta^{2}\left\langle\delta \Delta E^{2}\right\rangle_{e} / 2}
\end{aligned}
$$

The denominator in Eq. (13) can be easily evaluated with the assumption of Gaussian statistics for $\delta \Delta E$. The average $\left\langle e^{\beta \delta \Delta E}\right\rangle_{e}$ is the characteristic function, evaluated at $-i \beta$, for the probability distribution for $\delta \Delta E$, which if $\delta \Delta E$ is assumed to be a Gaussian random variable, is given by ${ }^{5}$

$$
\left\langle e^{\beta \delta \Delta E}\right\rangle_{e} \simeq e^{\beta^{2}\left\langle(\delta \Delta E)^{2}\right\rangle_{e} / 2} .
$$

Then, combining the two results, we find that assuming Gaussian statistics gives the approximation

$$
\langle\Delta E\rangle_{\mathrm{ne}}(t)-\langle\Delta E\rangle_{\mathrm{ne}}(\infty) \simeq \beta\langle\delta \Delta E(t) \delta \Delta E(0)\rangle_{e},
$$

and the resulting approximation to the normalized dynamics Stokes shift is the same as given by Eq. (16). Thus, the approximation of $S(t)$ by $C_{e}(t)$ is obtained by assuming that $\delta \Delta E$ is a Gaussian random process. It is not necessary to linearize $e^{\beta \delta \Delta E}$ or assume $\beta \delta \Delta E \ll 1$, nor is it necessary to use Eq. (8) and assume that the ground- and excited-state Gaussian statistics are the same to obtain Eq. (16).

It is interesting to examine why the linearization of $e^{\beta \delta \Delta E}$ yields the same result as the more general assumption of Gaussian statistics in the excited state. Note that while higher-order correlation functions, $\left\langle\delta \Delta E(t) \delta \Delta E(0)^{n}\right\rangle_{e}$ for $n \geqslant 2$, are neglected in the linearization, they are included in the Gaussian statistics approximation but they provide no new information, i.e., they are either zero or proportional to $\langle\delta \Delta E(t) \delta \Delta E(0)\rangle_{e}$. This is analogous to the neglect of the higher-order cumulants in the traditional linear-response approach. ${ }^{16,17}$ Importantly, this provides a straightforward way to check the validity of the approximation by comparing the higher-order correlation functions, $\left\langle\delta \Delta E(t) \delta \Delta E(0)^{n}\right\rangle_{e}$ for $n>2$, to their Wick's theorem factorizations. While expressions for the lowest-order neglected terms were obtained by Bernard and Callen ${ }^{16}$ for the traditional linear-response approach, their use as tests of the approximation has been extremely rare (see Ref. 17). Yet, they should represent a powerful diagnostic tool for both ground- and excited-state approaches.

Further, the present result shows that the excited-state correlation functions [Eqs. (16) and (24)] can be valid even when $\beta \delta \Delta E>1$, as is often the case; this indicates that, as long as the statistics are Gaussian, these approximations can be accurate even if configurations far from equilibrium are sampled. Related to this, we note that the linearization of $e^{\beta \delta \Delta E}$ implies a local approximation, i.e., that $\delta \Delta E$ is small, whereas the approximation used above to obtain Eq. (24) involves only the assumption that $\delta \Delta E$ exhibits globally averaged Gaussian statistics. Geissler and Chandler found a breakdown of the linear-response approximation in a system for which $C_{g}(t)=C_{e}(t)$ and for which they observed Gaussian statistics. ${ }^{21}$ They attributed the inequivalence of $S(t)$ and $C_{g}(t)=C_{e}(t)$ to the nonstationarity of the Gaussian statistics. The connection between the present approach and nonstationary Gaussian statistics requires further study (note that, in principle, Wick's theorem requires only that the statistics be Gaussian, not stationary).

Finally, the requirement of Gaussian statistics for $\delta \Delta E$ in the excited state is presumably a weaker one than that required for the validity of the traditional linear-response approximation [Eq. (8)], which assumes that the excited-state dynamics are not far removed from the ground-state dynamics. ${ }^{8,13,18}$ This implies that the approximation may have a wider validity for complex systems-e.g., timedependent fluorescence in nanoconfined solvents where the ground- and excited-state statistics can be dramatically different due to the dependence of the solute position on the electronic state - than indicated by the traditional linearresponse approximation.

It is noteworthy that other quantities besides the timedependent Stokes shift can be obtained using the Gaussian statistics approximation. Consider a variable $A$ which, for convenience, is assumed to have the property $\langle A\rangle_{e}=0$. Then, using the same approach as above, it is straightforward to show that

$$
\langle A\rangle_{\mathrm{ne}}(t) \simeq \beta\langle A(t) \delta \Delta E(0)\rangle_{e},
$$

assuming only Gaussian statistics. It is possible that such an approximation may be reasonable even when $A$ itself is not a Gaussian random process but $\delta \Delta E$ is. At present it is unclear if this is the case, and we are currently investigating this issue.

B.B.L. gratefully acknowledges support from the National Science Foundation under Grant No. CHE0316127. W.H.T. thanks the Chemical Sciences, Geosciences and Biosciences Division, Office of Basic Energy Sciences, Office of Science, U.S. Department of Energy for the support of this work.

${ }^{1}$ W. R. Ware, S. K. Lee, G. J. Brant, and P. P. Chow, J. Chem. Phys. 54, 4729 (1971); S. K. Chakrabarti and W. R. Ware, ibid. 55, 5494 (1971).

${ }^{2}$ E. W. Castner, Jr., M. Maroncelli, and G. R. Fleming, J. Chem. Phys. 86, 1090 (1987); M. Maroncelli and G. R. Fleming, ibid. 86, 6221 (1987).

${ }^{3}$ V. Nagarajan, A. M. Brearley, T.-J. Kang, and P. F. Barbara, J. Chem. Phys. 86, 3183 (1987).

${ }_{5}^{4}$ S.-G. Su and J. D. Simon, J. Phys. Chem. 91, 2693 (1987).

${ }^{5}$ R. Kubo, M. Toda, and N. Hashitsume, Statistical Physics II: Nonequilibrium Statistical Mechanics (Springer-Verlag, New York, 1978).

${ }^{6}$ See, e.g., M. Maroncelli and G. R. Fleming, J. Chem. Phys. 89, 5044 (1988).

${ }^{7}$ A. Nitzan, Chemical Dynamics in Condensed Phases (Oxford, New York, 2006)

${ }^{8}$ E. A. Carter and J. T. Hynes, J. Chem. Phys. 94, 5961 (1991).

${ }^{9}$ T. Fonseca and B. M. Ladanyi, J. Phys. Chem. 95, 2116 (1991); T. Fonseca and B. M. Ladanyi, J. Mol. Liq. 60, 1 (1994).

${ }^{10}$ L. Turi, P. Mináry, and P. J. Rossky, Chem. Phys. Lett. 316, 465 (2000).

${ }^{11}$ W. H. Thompson, J. Chem. Phys. 120, 8125 (2004). 
${ }^{12}$ M. J. Bedard-Hearn, R. E. Larsen, and B. J. Schwartz, J. Phys. Chem. A 107, 4773 (2003); J. Phys. Chem. B 107, 14464 (2003).

${ }^{13}$ G. Tao and R. M. Stratt, J. Chem. Phys. 125, 114501 (2006).

${ }^{14}$ M. S. Skaf and B. M. Ladanyi, J. Phys. Chem. 100, 18258 (1996).

${ }^{15}$ L. Onsager, Phys. Rev. 37, 405 (1931); 38, 2265 (1931).

${ }^{16}$ W. Bernard and H. B. Callen, Rev. Mod. Phys. 31, 1017 (1959) and references therein.
${ }^{17}$ A. A. Mosyak, O. V. Prezhdo, and P. J. Rossky, J. Chem. Phys. 109, 6390 (1998).

${ }^{18}$ D. Chandler, Introduction to Modern Statistical Mechanics (Oxford, New York, 1987), pp. 252-255.

${ }^{19}$ M. C. Wang and G. E. Uhlenbeck, Rev. Mod. Phys. 17, 323 (1945).

${ }^{20}$ G. C. Wick, Phys. Rev. 80, 268 (1950).

${ }^{21}$ P. L. Geissler and D. Chandler, J. Chem. Phys. 113, 9759 (2000). 